\title{
Identificación del consumo de alcohol y drogas en la Escuela Superior de Atotonilco de Tula
}

\author{
Identification of alcohol and drug consumption in the High School of Atotonilco de Tula \\ Gelacio Guzmán-Díaz ${ }^{a}$, Esmeralda Andrade-Tapia ${ }^{b}$, Jennifer Verdi-Estrada ${ }^{c}$
}

\begin{abstract}
:
A descriptive research was carried out to identify the level of consumption of alcoholic beverages and drugs in the Higher School of Atotonilco de Tula of the Autonomous University of the State of Hidalgo. 150 students participated in the educational programs of high school, Market Intelligence and Psychology. For the collection of data, the AUDIT and DAST-10 tests used for the identification of alcohol and drug consumption and frequency respectively were used. Likewise, three questions were asked to the respondents to complete the data obtained. The results are discussed around the relevance of the study and the scope of it.
\end{abstract}

\section{Keywords:}

Descriptive research, Addicitions, AUDIT, DAST-10, young

\section{Resumen:}

Se llevó a cabo una investigación de tipo descriptiva para identificar el nivel de consumo de bebidas alcohólicas y de drogas en la Escuela Superior de Atotonilco de Tula de la Universidad Autónoma del Estado de Hidalgo. Participaron 150 estudiantes de los programas educativos de bachillerato, licenciatura en Inteligencia de Mercados y Psicología. Para la recolección de datos se utilizaron los test AUDIT y DAST-10 utilizados para la identificación de consumo y frecuencia de alcohol y drogas respectivamente. Así mismo, se realizaron tres preguntas a los encuestados para completar los datos obtenidos. Los resultados se discuten en torno a la pertinencia del estudio y de los alcances del mismo.

\section{Palabras Clave:}

Investigación descriptiva, Adicciones, AUDIT, DAST-10, Jóvenes.

\section{Introducción}

El consumo de bebidas alcohólicas en México, se ha consolidado dentro de las costumbres sociales. En los últimos años se ha observado una tolerancia para el consumo riesgoso de alcohol, sobre todo cuando el abuso está ligado a situaciones de fiesta y diversión. Para algunos jóvenes y adolescentes, experimentar el consumo de alcohol puede ser un rito necesario para atravesar la fase de individuación. Sin embargo, para aquellos jóvenes y adolescentes que presentan personalidades complejas, puede constituir la única alternativa para enfrentar los problemas personales, y ser así los más sensibles a la dependencia de alcohol(1). La amplia aceptación social del consumo de alcohol facilita el consumo, en la población mexicana, incluso el inicio se da y se promueve en el hogar. Esto ocasiona que la percepción de riesgo sea baja, aspecto que ha llevado a que más del $70 \%$ de la población haya consumido alcohol. Además, en las cercanías de las escuelas, particularmente en aquellas de nivel bachillerato $y$ universitario, existe un alto ofrecimiento de alcohol en tiendas y expendios (2).

De acuerdo con el Instituto Nacional de Psiquiatría Ramón de la Fuente desde hace más de cuatro décadas, el consumo de alcohol es el problema más extendido en México, teniendo graves repercusiones sociales y de salud (2). Asimismo, el problema mundial de las drogas representa uno de los mayores desafíos para el país, por lo que resulta necesario conocer la frecuencia en que las drogas son consumidas, si son legales o ilegales, la proporción de hombres y mujeres que utilizan drogas con

\footnotetext{
a Autor de Correspondencia, Universidad Autónoma del Estado de Hidalgo, Escuela Superior de Atotonilco de Tula, ORCID: 0000-00034869-8658, Email: gelacio_guzman@uaeh.edu.mx 
fines de intoxicación, así como las entidades federativas con mayores índices de uso, abuso y dependencia (3). En México, el consumo de alcohol, tabaco y otras drogas en adolescentes ha sido objeto de varios estudios, debido a que en los últimos años se ha observado un incremento progresivo de la incidencia de consumo, que se inicia a edades cada vez más tempranas. Pese a los datos de aumento de ingesta de alcohol, sigue habiendo un bajo nivel de percepción de riesgo y alta tolerancia social ante su uso; asimismo, es importante mencionar que el inicio temprano en el consumo de alcohol incrementa en gran medida las probabilidades de consumir drogas ilegales (4).

De acuerdo con las cifras de la Encuesta Nacional de Consumo de Drogas, Alcohol y Tabaco 2016-2017, en la población total nacional el consumo en el último año de cualquier droga es de $10.3 \%$, drogas ilegales $9.9 \%$ y mariguana $8.6 \%$. La edad promedio en el que se inicia el consumo de drogas es de 17.8 años (3). Con respecto al alcohol, la prevalencia de consumo de alcohol es de $49 \%$ del total de la población. La edad promedio en el que se inicia el consumo de alcohol es de 17.9 años. Un dato relevante es que la Ciudad de México y el Estado de Hidalgo tienen los porcentajes más bajos de dependencia al alcohol con respecto a la media nacional (2).

En la población mexicana, el consumo de drogas aún es bajo en comparación con otros países, sin embargo, se mantiene en un crecimiento sostenido (3). En lo que respecta al alcohol, los resultados indican que en México el consumo es alto y con un patrón de consumo excesivo. Dicho patrón ha presentado un mayor crecimiento en menores de edad, sobretodo en mujeres (2).

Conocer los datos nacionales nos brinda un panorama general del consumo tanto de alcohol como de drogas, sin embargo, resulta importante obtener datos a nivel local para estar en condiciones de proponer estrategias de prevención y sobretodo intervención en caso de ser necesarios. Para realizar esta pesquisa, es necesario contar con instrumentos válidos y confiables. Dos de estos instrumentos son el Alcohol Use Disorders Identications Test (AUDIT) y el Drug Abuse Screening Test (DAST-10), ambos instrumentos han sido utilizados y probados en distintas investigaciones relacionadas con el consumo de alcohol y drogas.

Gómez-Maqueo, Gómez, Morales y Pérez (4) evaluaron la confiabilidad y validez en población mexicana del DAST-10 para detectar abuso de sustancias psicoactivas y del AUDIT para el abuso de alcohol. Ambas escalas se aplicaron a 915 alumnos de un bachillerato de la Ciudad de México. De acuerdo con los autores, los dos instrumentos resultaron válidos y confiables. Además, fueron sensibles en la identificación del riesgo en consumo de sustancias y alcohol al diferenciar niveles de riesgo entre hombres y mujeres.

EI AUDIT es una prueba que contiene 10 preguntas que identifican el uso de alcohol en los últimos 12 meses. Las tres primeras preguntas son en relación al consumo, las preguntas de 4 a 6 examinan síntomas de la dependencia del alcohol, mientras que las preguntas 7 a 10 exploran las consecuencias negativas asociadas a su consumo. Cada pregunta del AUDIT tiene de tres a cinco posibles respuestas que suman al final un máximo posible de 40 puntos. Puntuación entre 0 a 7 es indicador de abstemios o bajo riesgo, de 8 a 12 uso de riesgo o abuso y de 13 a 40 indica probable dependencia para los hombres.

EI DAST es un cuestionario con respuestas dicotómicas (Sí/No) diseñado para identificar sujetos con consumo problemático de drogas. Sus ítems integran cuestiones relativas a aspectos propios del uso abusivo de sustancias, así como a las complicaciones físicas, psicológicas y sociales que éste produce.

\section{Objetivo}

Identificar el nivel de consumo de alcohol y drogas de los estudiantes de la Escuela Superior de Atotonilco de Tula (ESAT) de la Universidad Autónoma del Estado de Hidalgo (UAEH).

\section{Método \\ Participantes}

La muestra estuvo conformada por 150 alumnos de la ESAT, con edades comprendidas entre $15-25$ años de edad. Participaron 50 estudiantes de Bachillerato (25 mujeres y 25 hombres), 50 alumnos de la licenciatura en Psicología (25 mujeres y 25 hombres) y 50 estudiantes de la licenciatura en Inteligencia de Mercados (14 hombres y 36 mujeres). La muestra se seleccionó de forma intencionada debido a que la población total de la licenciatura en Inteligencia de Mercados es de 65 alumnos, por lo que se decidió equiparar la muestra a 50 alumnos por programa educativo. Así mismo, este último programa sólo cuenta con 16 alumnos varones por lo que la muestra en este caso estuvo constituida por un mayor número de mujeres.

\section{Procedimiento}

Los participantes fueron seleccionados al azar y se les aplicaron los test AUDIT y DAST-10 en las zonas recreativas de la ESAT. La aplicación se llevó a cabo durante una semana por 6 estudiantes de cuarto semestre de la carrera de psicología. A cada participante se le entregaban los cuestionarios impresos y el aplicador le daba las instrucciones de llenado. La aplicación individual tuvo una duración aproximada de 10 minutos. A ninguno de los participantes se le pidió su nombre o cualquier otro dato de identificación, esto con el objetivo de mantener el anonimato y brindarles la seguridad y confianza de contestar las preguntas con la mayor veracidad posible. Al momento de la aplicación y para complementar la información de los dos instrumentos se realizaron tres preguntas abiertas: 1. ¿Consideras que tienes algún problema con el consumo de bebidas alcohólicas o drogas? ¿Por qué?, 2. ¿Consideras que en tu escuela hay un problema de consumo de bebidas alcohólicas y/o drogas? ¿Por qué?, 3. ¿Participarías en una investigación o tratamiento para disminuir el consumo de bebidas alcohólicas y/o drogas? ¿Por qué?

\section{Resultados}

A continuación, se presentan los resultados obtenidos durante la presente investigación. En la Tabla 1 se presentan los porcentajes obtenidos en cada una de las preguntas del AUDIT por los alumnos del programa educativo de bachillerato. En la pregunta 1. ¿Con qué frecuencia consumes alguna bebida alcohólica? La mayoría de los estudiantes contesto que nunca, sin 
embargo, más de la mitad de los estudiantes contesto que consumen bebidas alcohólicas de 1 a 4 veces al mes, este dato es relevante debido a que el grueso de esta población no tiene la edad legal para consumir este tipo de bebidas. En cuanto a la frecuencia de consumo no se observa en los encuestados una conducta de consumo que llegue a la embriaguez. Los porcentajes con respecto a la dependencia del alcohol son muy bajos y en cuanto a consecuencias negativas por el consumo parece sólo haber casos aislados. Es de destacar la pregunta 10. ¿Algún familiar, amigo o médico ha mostrado preocupación por tú consumo de bebidas alcohólicas o te ha sugerido que dejes de beber?, $16 \%$ de los encuestados contestaron afirmativa esta cuestión, esto sugiere que, aunque hay pocos casos de consumo frecuente los familiares de estos son sensibles a la situación.

Tabla 1. Porcentajes obtenidos en el AUDIT por los alumnos de Bachillerato-ESAT

\begin{tabular}{|c|c|c|c|c|c|}
\hline \multicolumn{6}{|c|}{ Bachillerato } \\
\hline Respuesta & $\begin{array}{c}\text { Nunc } \\
\text { a }\end{array}$ & $\begin{array}{l}\text { Men } \\
\text { os de } \\
\text { una } \\
\text { vez } \\
\text { al } \\
\text { mes }\end{array}$ & $\begin{array}{c}\text { De } 2 \\
\text { a } 4 \\
\text { vece } \\
\text { s al } \\
\text { mes }\end{array}$ & $\begin{array}{c}\text { De } 2 \\
\text { a } 3 \\
\text { más } \\
\text { veces } \\
\text { a la } \\
\text { sema } \\
\text { na }\end{array}$ & $\begin{array}{c}4 \text { o } \\
\text { más } \\
\text { veces } \\
\text { a la } \\
\text { sema } \\
\text { na }\end{array}$ \\
\hline $\begin{array}{l}\text { 1. ¿Con qué } \\
\text { frecuencia } \\
\text { consumes } \\
\text { alguna } \\
\text { bebida } \\
\text { alcohólica? }\end{array}$ & $42 \%$ & $24 \%$ & $32 \%$ & $2 \%$ & $0 \%$ \\
\hline $\begin{array}{l}\text { 2. ¿Cuantas } \\
\text { bebidas } \\
\text { alcohólicas } \\
\text { consumes } \\
\text { en un día en } \\
\text { el que } \\
\text { tomas? }\end{array}$ & $70 \%$ & $16 \%$ & $4 \%$ & $6 \%$ & $4 \%$ \\
\hline $\begin{array}{l}\text { 3. ¿Con qué } \\
\text { frecuencia } \\
\text { tomas } 5 \text { o } \\
\text { más } \\
\text { bebidas } \\
\text { alcohólicas } \\
\text { en un solo } \\
\text { día? }\end{array}$ & $72 \%$ & $18 \%$ & $4 \%$ & $6 \%$ & $0 \%$ \\
\hline $\begin{array}{l}\text { 4. ¿Con qué } \\
\text { frecuencia } \\
\text { en el curso } \\
\text { del último } \\
\text { año has } \\
\text { sido incapaz } \\
\text { de parar de } \\
\text { beber una } \\
\text { vez que has } \\
\text { empezado? }\end{array}$ & $92 \%$ & $6 \%$ & $0 \%$ & $2 \%$ & $0 \%$ \\
\hline $\begin{array}{l}\text { 5. ¿Con qué } \\
\text { frecuencia } \\
\text { en el curso }\end{array}$ & $94 \%$ & $6 \%$ & $0 \%$ & $0 \%$ & $0 \%$ \\
\hline
\end{tabular}

\begin{tabular}{|c|c|c|c|c|c|}
\hline $\begin{array}{l}\text { del último } \\
\text { año no } \\
\text { pudiste } \\
\text { realizar tus } \\
\text { actividades } \\
\text { porque } \\
\text { habías } \\
\text { bebido? }\end{array}$ & & & & & \\
\hline $\begin{array}{l}\text { 6. ¿Con qué } \\
\text { frecuencia } \\
\text { en el curso } \\
\text { del último } \\
\text { año has } \\
\text { necesitado } \\
\text { beber en } \\
\text { ayunas para } \\
\text { recuperarte } \\
\text { después de } \\
\text { haber } \\
\text { bebido } \\
\text { mucho el } \\
\text { día anterior? }\end{array}$ & $\begin{array}{c}100 \\
\%\end{array}$ & $0 \%$ & $0 \%$ & $0 \%$ & $0 \%$ \\
\hline $\begin{array}{l}\text { 7. ¿Con qué } \\
\text { frecuencia } \\
\text { en el curso } \\
\text { del último } \\
\text { año has } \\
\text { tenido } \\
\text { remordimien } \\
\text { tos o } \\
\text { sentimientos } \\
\text { de culpa } \\
\text { después de } \\
\text { haber } \\
\text { bebido? }\end{array}$ & $86 \%$ & $8 \%$ & $6 \%$ & $0 \%$ & $0 \%$ \\
\hline $\begin{array}{l}\text { 8. ¿Con qué } \\
\text { frecuencia } \\
\text { en el curso } \\
\text { del último } \\
\text { año no has } \\
\text { podido } \\
\text { recordar lo } \\
\text { que sucedió } \\
\text { la noche } \\
\text { anterior } \\
\text { porque } \\
\text { habías } \\
\text { estado } \\
\text { bebiendo? }\end{array}$ & $82 \%$ & $12 \%$ & $2 \%$ & $2 \%$ & $2 \%$ \\
\hline $\begin{array}{l}\text { 9. ¿Tú o } \\
\text { alguna otra } \\
\text { persona ha } \\
\text { resultado } \\
\text { herido } \\
\text { porque tú } \\
\text { habías } \\
\text { bebido? }\end{array}$ & $96 \%$ & $2 \%$ & $2 \%$ & $0 \%$ & $0 \%$ \\
\hline $\begin{array}{l}\text { 10. ¿Algún } \\
\text { familiar, } \\
\text { amigo o } \\
\text { médico ha } \\
\text { mostrado }\end{array}$ & $84 \%$ & $0 \%$ & $14 \%$ & $0 \%$ & $2 \%$ \\
\hline
\end{tabular}




\begin{tabular}{|l|l|l|l|l|}
\hline preocupació & & & & \\
$\mathrm{n}$ por tú & & & \\
consumo de & & & & \\
bebidas & & & \\
alcohólicas & & & \\
o te ha & & & \\
sugerido & & & \\
que dejes & & & & \\
de beber? & & & & \\
\hline
\end{tabular}

En la Tabla 2 se presentan los porcentajes obtenidos en cada una de las preguntas del AUDIT por los alumnos del programa educativo de licenciatura en Inteligencia de Mercados. Hay que recordar que la mayoría de esta población fueron mujeres pero que a comparación de los estudiantes de Bachillerato, a nivel licenciatura los alumnos tienen una edad legal para el consumo de bebidas alcohólicas. En la pregunta 1. ¿Con qué frecuencia consumes alguna bebida alcohólica? Cerca de la mitad de los encuestados, específicamente el $46 \%$, tiene un consumo mensual de 2 a 4 veces al mes, así mismo sólo el $10 \%$ respondió no beber nunca por lo que el $90 \%$ de los alumnos en Inteligencia de Mercados ha consumido bebidas alcohólicas en el último año. Con respecto al exceso de consumo, aunque es más elevado que el mostrado en nivel bachillerato, la distribución de los porcentajes no muestra consumo excesivo de bebidas alcoholicas en este sector de la población. Con respecto a las preguntas 7. ¿Con qué frecuencia en el curso del último año has tenido remordimientos o sentimientos de culpa después de haber bebido? Y 8. ¿Con qué frecuencia en el curso del último año no has podido recordar lo que sucedió la noche anterior porque habías estado bebiendo?, un cuarto de la población contesto haber tenido remordimiento o no haber recordado lo que sucedió después de una noche de consumo de alcohol, es importante considerar este dato, pues, aunque son pocos los casos, ambas situaciones plantean consecuencias negativas del consumo de alcohol que pudiesen desencadenar problemas personales 0 sociales.

Tabla 2. Porcentajes obtenidos en el AUDIT por los alumnos de Inteligencia de Mercados-ESAT

\begin{tabular}{|l|c|c|c|c|c|}
\hline \multicolumn{5}{|c|}{ Inteligencia de Mercados } \\
\hline Respuesta & $\begin{array}{c}\text { Nunc } \\
\text { a }\end{array}$ & $\begin{array}{c}\text { Men } \\
\text { os de } \\
\text { una } \\
\text { vez } \\
\text { al }\end{array}$ & $\begin{array}{c}\text { De } 2 \\
\text { a } 4 \\
\text { vece } \\
\text { s al } \\
\text { Mes }\end{array}$ & $\begin{array}{c}\text { De 2 } \\
\text { a 3 } \\
\text { más } \\
\text { veces } \\
\text { a la } \\
\text { sema } \\
\text { na }\end{array}$ & $\begin{array}{c}4 \text { o } \\
\text { más } \\
\text { veces } \\
\text { a la } \\
\text { sema } \\
\text { na }\end{array}$ \\
\hline $\begin{array}{l}1 . \text { ¿Con qué } \\
\text { frecuencia } \\
\text { consumes } \\
\text { alguna } \\
\text { bebida } \\
\text { alcohólica? }\end{array}$ & $10 \%$ & $30 \%$ & $46 \%$ & $14 \%$ & $0 \%$ \\
\hline $\begin{array}{l}2 . \text { ¿Cuantas } \\
\text { bebidas } \\
\text { alcohólicas } \\
\text { consumes }\end{array}$ & $54 \%$ & $18 \%$ & $10 \%$ & $10 \%$ & $8 \%$ \\
\hline
\end{tabular}

\begin{tabular}{|c|c|c|c|c|c|}
\hline $\begin{array}{l}\text { en un día en } \\
\text { el que } \\
\text { tomas? }\end{array}$ & & & & & \\
\hline $\begin{array}{l}\text { 3. ¿Con qué } \\
\text { frecuencia } \\
\text { tomas } 5 \text { o } \\
\text { más } \\
\text { bebidas } \\
\text { alcohólicas } \\
\text { en un solo } \\
\text { día? }\end{array}$ & $48 \%$ & $26 \%$ & $18 \%$ & $8 \%$ & $0 \%$ \\
\hline $\begin{array}{l}\text { 4. ¿Con qué } \\
\text { frecuencia } \\
\text { en el curso } \\
\text { del último } \\
\text { año has } \\
\text { sido incapaz } \\
\text { de parar de } \\
\text { beber una } \\
\text { vez que has } \\
\text { empezado? }\end{array}$ & $70 \%$ & $18 \%$ & $6 \%$ & $6 \%$ & $0 \%$ \\
\hline $\begin{array}{l}\text { 5. ¿Con qué } \\
\text { frecuencia } \\
\text { en el curso } \\
\text { del último } \\
\text { año no } \\
\text { pudiste } \\
\text { realizar tus } \\
\text { actividades } \\
\text { porque } \\
\text { habías } \\
\text { bebido? }\end{array}$ & $82 \%$ & $14 \%$ & $2 \%$ & $2 \%$ & $0 \%$ \\
\hline $\begin{array}{l}\text { 6. ¿Con qué } \\
\text { frecuencia } \\
\text { en el curso } \\
\text { del último } \\
\text { año has } \\
\text { necesitado } \\
\text { beber en } \\
\text { ayunas para } \\
\text { recuperarte } \\
\text { después de } \\
\text { haber } \\
\text { bebido } \\
\text { mucho el } \\
\text { día anterior? }\end{array}$ & $86 \%$ & $6 \%$ & $6 \%$ & $2 \%$ & $0 \%$ \\
\hline $\begin{array}{l}\text { 7. ¿Con qué } \\
\text { frecuencia } \\
\text { en el curso } \\
\text { del último } \\
\text { año has } \\
\text { tenido } \\
\text { remordimien } \\
\text { tos o } \\
\text { sentimientos } \\
\text { de culpa } \\
\text { después de } \\
\text { haber } \\
\text { bebido? }\end{array}$ & $70 \%$ & $24 \%$ & $4 \%$ & $2 \%$ & $0 \%$ \\
\hline $\begin{array}{l}\text { 8. ¿Con qué } \\
\text { frecuencia } \\
\text { en el curso }\end{array}$ & $72 \%$ & $22 \%$ & $4 \%$ & $2 \%$ & $0 \%$ \\
\hline
\end{tabular}




\begin{tabular}{|c|c|c|c|c|c|}
\hline $\begin{array}{l}\text { del último } \\
\text { año no has } \\
\text { podido } \\
\text { recordar lo } \\
\text { que sucedió } \\
\text { la noche } \\
\text { anterior } \\
\text { porque } \\
\text { habías } \\
\text { estado } \\
\text { bebiendo? }\end{array}$ & & & & & \\
\hline $\begin{array}{l}\text { 9. ¿Tú o } \\
\text { alguna otra } \\
\text { persona ha } \\
\text { resultado } \\
\text { herido } \\
\text { porque tú } \\
\text { habías } \\
\text { bebido? }\end{array}$ & $92 \%$ & $0 \%$ & $4 \%$ & $0 \%$ & $4 \%$ \\
\hline $\begin{array}{l}\text { 10. ¿Algún } \\
\text { familiar, } \\
\text { amigo o } \\
\text { médico ha } \\
\text { mostrado } \\
\text { preocupació } \\
\text { n por tú } \\
\text { consumo de } \\
\text { bebidas } \\
\text { alcohólicas } \\
\text { o te ha } \\
\text { sugerido } \\
\text { que dejes } \\
\text { de beber? }\end{array}$ & $78 \%$ & $0 \%$ & $12 \%$ & $0 \%$ & $10 \%$ \\
\hline
\end{tabular}

En la Tabla 3 se presentan los porcentajes obtenidos en cada una de las preguntas del AUDIT por los alumnos del programa educativo de licenciatura en Psicología. Al igual que los encuestados anteriores, lo alumnos de Psicología tienen una edad legal para el consumo de bebidas alcohólicas. Por otra parte, a diferencia de la licenciatura en Inteligencia de Mercados, la población total de alumnos de Psicología es cinco veces más grande, por lo que la convivencia y socialización es más variada. En la pregunta 1. ¿Con qué frecuencia consumes alguna bebida alcohólica? al igual que en el caso anterior el $90 \%$ de los encuestados ha consumido bebidas embriagantes en el último año. El consumo no varía mucho en comparación con la licenciatura en Inteligencia de Mercados, en ambos casos resulta más frecuente y excesivo en comparación con Bachillerato, pero si llegar a los niveles extremos. Al igual que en los casos anteriores, las preguntas relacionadas con las consecuencias negativas derivas de la ingesta de alcohol tiene porcentajes significativos alrededor de un cuarto de la población encuestada, es de destacar este dato debido a que el alcoholismo se considera un problema social a parir de este tipo de consecuencias.

Tabla 3. Porcentajes obtenidos en el AUDIT por los alumnos de Psicología-ESAT Psicología

\begin{tabular}{|c|c|c|c|c|c|}
\hline Respuesta & $\begin{array}{c}\text { Nunc } \\
\text { a }\end{array}$ & $\begin{array}{c}\text { Men } \\
\text { os de } \\
\text { una } \\
\text { vez } \\
\text { al } \\
\text { mes }\end{array}$ & $\begin{array}{c}\text { De } 2 \\
\text { a } 4 \\
\text { vece } \\
\text { s al } \\
\text { Mes }\end{array}$ & $\begin{array}{c}\text { De } 2 \\
\text { a } 3 \\
\text { más } \\
\text { veces } \\
\text { a la } \\
\text { sema } \\
\text { na }\end{array}$ & $\begin{array}{c}4 \text { o } \\
\text { más } \\
\text { veces } \\
\text { a la } \\
\text { sema } \\
\text { na }\end{array}$ \\
\hline $\begin{array}{l}\text { 1. ¿Con qué } \\
\text { frecuencia } \\
\text { consumes } \\
\text { alguna } \\
\text { bebida } \\
\text { alcohólica? }\end{array}$ & $10 \%$ & $26 \%$ & $50 \%$ & $14 \%$ & $0 \%$ \\
\hline $\begin{array}{l}\text { 2. ¿Cuantas } \\
\text { bebidas } \\
\text { alcohólicas } \\
\text { consumes } \\
\text { en un día en } \\
\text { el que } \\
\text { tomas? }\end{array}$ & $36 \%$ & $32 \%$ & $16 \%$ & $8 \%$ & $8 \%$ \\
\hline $\begin{array}{l}\text { 3. ¿Con qué } \\
\text { frecuencia } \\
\text { tomas } 5 \text { o } \\
\text { más } \\
\text { bebidas } \\
\text { alcohólicas } \\
\text { en un solo } \\
\text { día? }\end{array}$ & $34 \%$ & $26 \%$ & $18 \%$ & $22 \%$ & $0 \%$ \\
\hline $\begin{array}{l}\text { 4. ¿Con qué } \\
\text { frecuencia } \\
\text { en el curso } \\
\text { del último } \\
\text { año has } \\
\text { sido incapaz } \\
\text { de parar de } \\
\text { beber una } \\
\text { vez que has } \\
\text { empezado? }\end{array}$ & $58 \%$ & $18 \%$ & $16 \%$ & $8 \%$ & $0 \%$ \\
\hline $\begin{array}{l}\text { 5. ¿Con qué } \\
\text { frecuencia } \\
\text { en el curso } \\
\text { del último } \\
\text { año no } \\
\text { pudiste } \\
\text { realizar tus } \\
\text { actividades } \\
\text { porque } \\
\text { habías } \\
\text { bebido? }\end{array}$ & $72 \%$ & $20 \%$ & $6 \%$ & $2 \%$ & $0 \%$ \\
\hline $\begin{array}{l}\text { 6. ¿Con qué } \\
\text { frecuencia } \\
\text { en el curso } \\
\text { del último } \\
\text { año has } \\
\text { necesitado } \\
\text { beber en } \\
\text { ayunas para } \\
\text { recuperarte } \\
\text { después de } \\
\text { haber } \\
\text { bebido }\end{array}$ & $76 \%$ & $12 \%$ & $10 \%$ & $2 \%$ & $0 \%$ \\
\hline
\end{tabular}




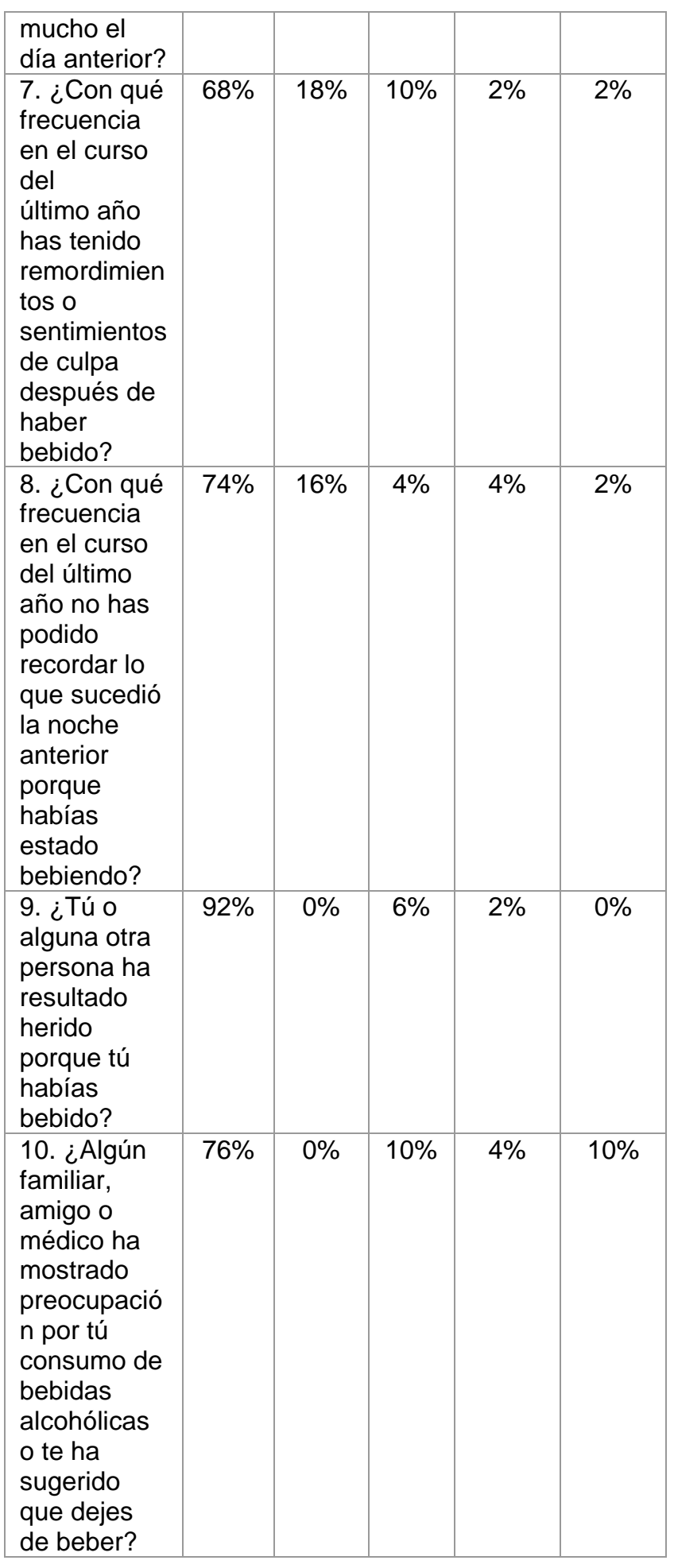

En la Tabla 4 se presentan los porcentajes obtenidos en cada una de las preguntas del DAST-10 por los alumnos de cada uno de los programas educativos que conforman la ESAT. Recordemos que este instrumento identifica el consumo de drogas sin incluir el alcohol. Los resultados son acordes con las con las estadísticas nacionales en el sentido de que el consumo de alcohol en jóvenes supera el consumo de drogas. En el caso de bachillerato más del $90 \%$ de los encuestados no ha consumido algún tipo de droga, en el caso los programas de licenciatura el consumo de este tipo de sustancias es más elevado, cerca de un cuarto de la población en ambas carreras ha consumido algún tipo de droga. Debido al bajo consumo presentado por los estudiantes encuestado el resto de las preguntas del DAST-10 en su mayoría fueron contestadas de forma negativa. Vale la pena mencionar que en la pregunta 7. ¿Ha abandonado a su familia por su uso de las drogas? ninguno de los encuantados contestó afirmativamente, por lo que podemos concluir que en el aspecto familiar, el consumo de drogas que dicen haber tenido algunos estudiantes no ha tenido un impacto negativo en sus familias. Con respecto a la pregunta 8. ¿Ha participado en actividades ilegales para obtener drogas? 4\% de la licenciatura en Inteligencia de Mercados y $8 \%$ de la carrera de Psicología respondieron afirmativamente a este cuestionamiento, aun cuando son pocos los casos parece relevante considerarlo debido a que dichos actos pudiesen derivar en consecuencias legales.

Tabla 4. Porcentajes obtenidos en el DAST-10 por los alumnos de la ESAT

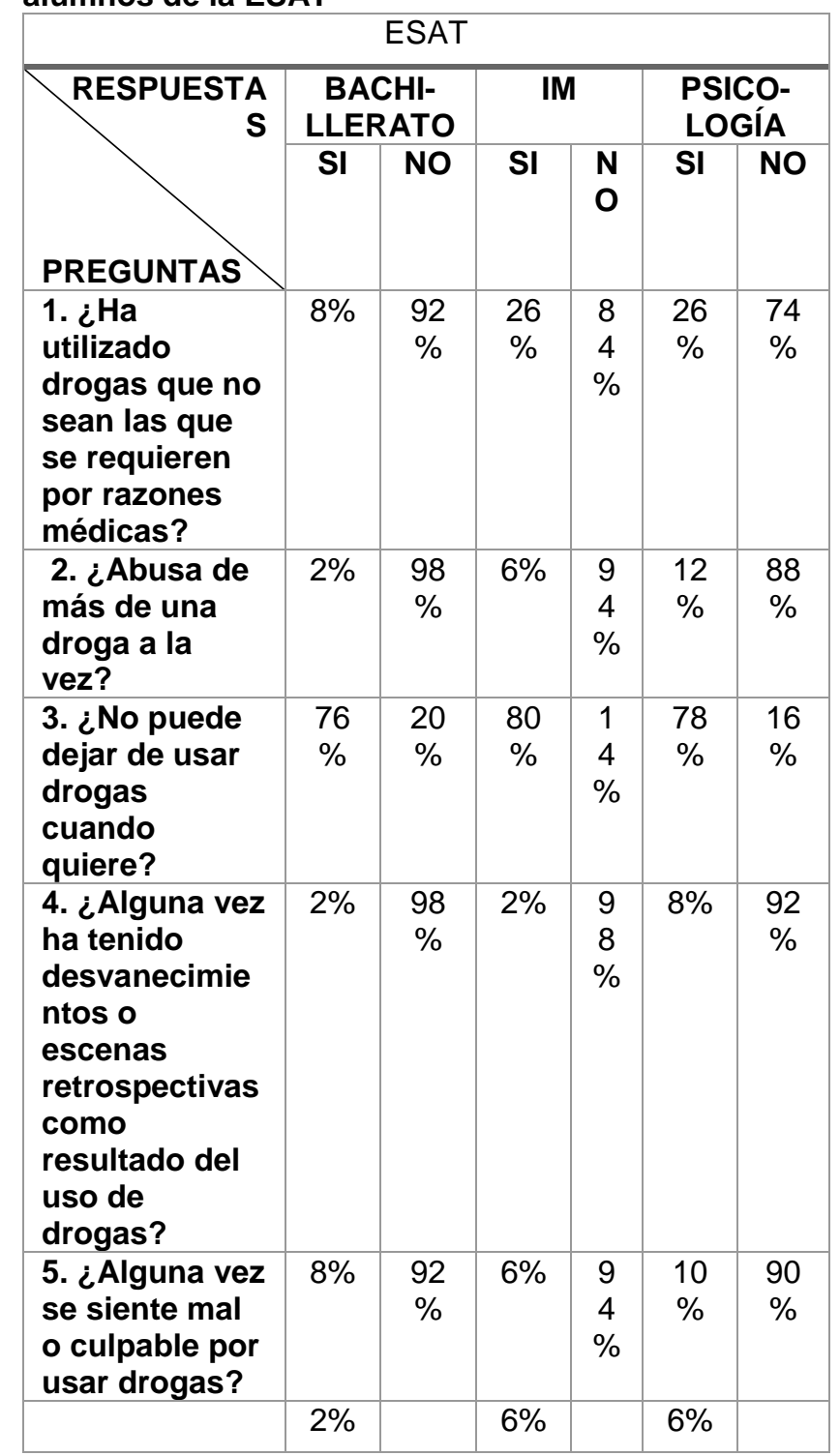




\begin{tabular}{|c|c|c|c|c|c|c|}
\hline $\begin{array}{l}\text { 6. ¿Su } \\
\text { cónyuge (o } \\
\text { padres) se } \\
\text { queja alguna } \\
\text { vez por su } \\
\text { participación } \\
\text { con las } \\
\text { drogas? }\end{array}$ & & $\begin{array}{l}98 \\
\%\end{array}$ & & $\begin{array}{l}9 \\
2 \\
\%\end{array}$ & & $\begin{array}{l}94 \\
\%\end{array}$ \\
\hline $\begin{array}{l}\text { 7. ¿Ha } \\
\text { abandonado a } \\
\text { su familia por } \\
\text { su uso de las } \\
\text { drogas? }\end{array}$ & $0 \%$ & $\begin{array}{c}100 \\
\%\end{array}$ & $0 \%$ & $\begin{array}{l}1 \\
0 \\
0 \\
\%\end{array}$ & $0 \%$ & $\begin{array}{c}100 \\
\%\end{array}$ \\
\hline $\begin{array}{l}\text { 8. ¿Ha } \\
\text { participado en } \\
\text { actividades } \\
\text { ilegales para } \\
\text { obtener } \\
\text { drogas? }\end{array}$ & $0 \%$ & $\begin{array}{c}100 \\
\%\end{array}$ & $4 \%$ & $\begin{array}{l}9 \\
6 \\
\%\end{array}$ & $8 \%$ & $\begin{array}{l}90 \\
\%\end{array}$ \\
\hline $\begin{array}{l}\text { 9. ¿Alguna vez } \\
\text { ha } \\
\text { experimentad } \\
\text { o síntomas de } \\
\text { retraimiento } \\
\text { (se ha sentido } \\
\text { enfermo) } \\
\text { cuando dejó } \\
\text { de tomar } \\
\text { drogas? }\end{array}$ & $0 \%$ & $\begin{array}{c}100 \\
\%\end{array}$ & $0 \%$ & $\begin{array}{l}1 \\
0 \\
0 \\
\%\end{array}$ & $2 \%$ & $\begin{array}{l}98 \\
\%\end{array}$ \\
\hline $\begin{array}{l}\text { 10. ¿Ha tenido } \\
\text { problemas } \\
\text { médicos como } \\
\text { resultado de } \\
\text { su uso de las } \\
\text { drogas (por } \\
\text { ejemplo, } \\
\text { pérdida de } \\
\text { memoria, } \\
\text { hepatitis, } \\
\text { convulsiones, } \\
\text { sangrado)? }\end{array}$ & $0 \%$ & $\begin{array}{c}100 \\
\%\end{array}$ & $2 \%$ & $\begin{array}{l}9 \\
8 \\
\%\end{array}$ & $0 \%$ & $\begin{array}{c}100 \\
\%\end{array}$ \\
\hline
\end{tabular}

En la Tabla 5 se presenta la distribución por zonas de riesgo de acuerdo con el AUDIT de los alumnos de la ESAT. A diferencia de los datos anteriores, estos no se presentan en porcentajes sino en frecuencias, debido a que resulta relevante conocer el número de casos particulares que posiblemente requieran atención. La mayoría de los encuestados, específicamente 106 alumnos se encuentran en la zona I, es decir, con un bajo consumo de alcohol. 33 alumnos se encuentran en la zona II, que no es una zona de riesgo. Las zonas III y IV corresponde a consumidores con riesgo y con posible dependencia al alcohol, en estos casos, únicamente un alumnos de bachillerato se encuentra en la zona III, pero 10 alumnos de licenciatura están ubicados en estas zonas.

Tabla 5. Distribución por zonas de riesgo de acuerdo con el AUDIT de los alumnos de la ESAT.

AUDIT: Zonas de riego

\begin{tabular}{|l|c|c|c|c|}
\hline & I & II & III & IV \\
\hline Bachillerato & 41 & 8 & 1 & 0 \\
\hline IM & 35 & 11 & 2 & 2 \\
\hline Psicología & 30 & 14 & 3 & 3 \\
\hline Total & 106 & 33 & 6 & 5 \\
\hline - & $\begin{array}{l}\text { Zona I (< 8). Bajo riesgo o abstinencia; sólo es } \\
\text { necesario una educación referente al alcohol. } \\
\text { Zona II (8 - 15). Consumo por sobre el bajo riesgo; } \\
\text { recomendado el consejo simple. } \\
\text { Zona III (16 - 19). Consumo de riesgo y perjudicial; } \\
\text { mediante un consejo simple, terapia breve o } \\
\text { monitorización continúa. } \\
\text { Zona IV (20 - 40). Posible dependencia al alcohol; } \\
\text { existe derivación al especialista para la evaluación } \\
\text { diagnóstica y tratamiento. }\end{array}$ \\
\hline
\end{tabular}

En la Tabla 6 se presenta la distribución por zonas de riesgo de acuerdo con el DAST-10 de los alumnos de la ESAT. Nuevamente se presentan frecuencias en esta tabla para hacer más discriminables los casos relevantes. En el caso del DAST-10 la pregunta 1. ¿Ha utilizado drogas que no sean las que se requieren por razones médicas? En la que una cuarta parte de cada una de las licenciaturas contestó afirmativamente coloca a 106 personas en la zona II de riesgo. Así mismo el ítem 3. ¿No puede dejar de usar drogas cuando quiere? Presenta un cierto sesgo debido a la redacción negativa de la pregunta, responder afirmativamente equivale a contestar que SI consumo pero lo puedo dejar. Ambas preguntas influyen para colocar a gran número de estudiantes en las zonas II y III cuando en realidad podría ser un efecto de lo ya mencionado. Sin embargo habría que señalar que cuando menos se reportaron dos casos en la zona IV de la escala, es decir, estos estudiantes pudiesen tener una demencia a la drogas.

Tabla 6. Distribución por zonas de riesgo de acuerdo con el DAST-10 de los alumnos de la ESAT.

\begin{tabular}{|l|c|c|c|c|}
\hline \multirow{2}{*}{ Programa Educativo } & \multicolumn{4}{|c|}{ DAST-10: Zonas de riego } \\
\cline { 2 - 5 } & I & II & III & IV \\
\hline Bachillerato & 6 & 41 & 3 & 0 \\
\hline IM & 9 & 35 & 5 & 1 \\
\hline Psicología & 10 & 30 & 9 & 1 \\
\hline Total & 25 & 106 & 17 & 2 \\
\hline - Zona I (0) - Bajo riesgo / abstinencia \\
- Zona II (1 - 2) - Riesgo \\
- Zona III (3 - 5) - Perjudicial \\
- Zona IV (6 +) - Dependiente \\
\hline
\end{tabular}

En la Tabla 6 se presenta los porcentajes de respuesta de los alumnos de la ESAT con respecto a las preguntas abiertas realizadas junto con el AUDIT y el DAST-10. Estas preguntas se consideraron importantes debido a 
que se esperaba conocer la percepción del estudiante con respecto a su consumo y al de sus pares independientemente de los resultados obtenidos en las escalas anteriores. Los resultados muestran que el $92 \%$ de la población en cada estrato no considera tener un problema con el consumo de drogas y alcohol, es relevante este dato puesto que aunque se observó en los instrumentos utilizados que si hay un consumo considerable los estudiantes no lo consideran como un problema. Con respecto a la segunda pregunta, los resultados son muy parecidos en cada uno de los programas educativos, la mitad de los estudiantes considera que hay un problema con el consumo de alcohol y/o drogas en sus compañeros. Finalmente, un resultado que vale la pena señalar es con respecto a la participación de los encuestados en investigación o tratamiento para la disminución del consumo de alcohol y/o drogas que presentan actual que tienen, en cada caso más de la mitad respondió afirmativamente a la pregunta, destacando que el $82 \%$ de la población de psicología participaría.

Tabla 7. Porcentaje de respuestas por los alumnos de la ESAT a las preguntas abiertas.

\begin{tabular}{|c|c|c|c|c|c|c|}
\hline & \multicolumn{2}{|c|}{$\begin{array}{l}\text { Bachi- } \\
\text { Ilerato }\end{array}$} & \multicolumn{2}{|c|}{ IM } & \multicolumn{2}{|c|}{$\begin{array}{l}\text { Psico- } \\
\text { logía }\end{array}$} \\
\hline $\begin{array}{l}\text { Respuestas } \\
\text { Preguntas }\end{array}$ & SI & $\mathrm{NO}$ & SI & NO & SI & $\begin{array}{l}\mathrm{N} \\
\mathrm{O}\end{array}$ \\
\hline $\begin{array}{l}\text { ¿Consideras que } \\
\text { tienes algún } \\
\text { problema con el } \\
\text { consumo } \\
\text { bebidas } \\
\text { alcohólicas } \\
\text { drogas? }\end{array}$ & $8 \%$ & $\begin{array}{l}92 \\
\%\end{array}$ & $8 \%$ & $\begin{array}{l}92 \\
\%\end{array}$ & $8 \%$ & $\begin{array}{l}92 \\
\%\end{array}$ \\
\hline $\begin{array}{lr}\text { ¿Consideras } & \text { que } \\
\text { en tu escuela hay } \\
\text { un problema } & \text { de } \\
\text { consumo } & \text { de } \\
\text { bebidas } & \\
\text { alcohólicas } & \text { y/o } \\
\text { drogas? } & \end{array}$ & $\begin{array}{l}50 \\
\%\end{array}$ & $\begin{array}{l}50 \\
\%\end{array}$ & $\begin{array}{l}40 \\
\%\end{array}$ & $\begin{array}{l}60 \\
\%\end{array}$ & $\begin{array}{l}54 \\
\%\end{array}$ & $\begin{array}{l}46 \\
\%\end{array}$ \\
\hline $\begin{array}{l}\text { ¿Participarías en } \\
\text { una investigación } \\
0 \quad \text { tratamiento } \\
\text { para disminuir el } \\
\text { consumo de } \\
\text { bebidas } \\
\text { alcohólicas y/o } \\
\text { drogas? }\end{array}$ & $\begin{array}{l}58 \\
\%\end{array}$ & $\begin{array}{l}42 \\
\%\end{array}$ & $\begin{array}{l}70 \\
\%\end{array}$ & $\begin{array}{l}30 \\
\%\end{array}$ & $\begin{array}{l}82 \\
\%\end{array}$ & $\begin{array}{l}18 \\
\%\end{array}$ \\
\hline
\end{tabular}

Finalmente cabe señalar que aun cuando los porcentajes podrían no mostrar niveles considerables de riesgo, los casos particulares no dejan de ser relevantes por lo que se considera importante, aun cuando sean pocos, los casos de estudiantes que pudiesen mostrar problemas de consumo excesivo o dependencia. A este respecto, fueron tres los casos en los que se obtuvieron resultados considerados como riesgosos en la presente investigación. Uno de ellos del sexo masculino, quien se situó en la zona IV del AUDIT y zona III del DAST, este sujeto respondió de la siguiente manera los cuestionamientos de las preguntas abiertas. 1. ¿Consideras que tienes algún problema con el consumo de bebidas alcohólicas o drogas? ¿Por qué? $R=$ Sí, porque tomo demasiado. 2. ¿Consideras que en tu escuela hay un problema de consumo de bebidas alcohólicas y/o drogas? ¿Por qué? Sí, porque sus alumnos toman demasiado, 3. ¿Participarías en una investigación o tratamiento para disminuir el consumo de bebidas alcohólicas y/o drogas? ¿Por qué? Sí, porque quiero disminuir mi consumo.

Otros dos casos similares al anterior se dieron en la carrera de psicología, el primero, una mujer quien se situó en la zona III en ambos instrumentos. Ella respondió a las preguntas abiertas de la siguiente manera 1. ¿Consideras que tienes algún problema con el consumo de bebidas alcohólicas o drogas? ¿Por qué? $R=$ No, puedo dejar de consumir si lo deseo. 2. ¿Consideras que en tu escuela hay un problema de consumo de bebidas alcohólicas y/o drogas? ¿Por qué? No, no sé sobre ello. 3. ¿Participarías en una investigación o tratamiento para disminuir el consumo de bebidas alcohólicas y/o drogas? ¿Por qué? Sí, porque tengo amigos con problemas de adicciones. El segundo caso en psicología corresponde a un varón situado en la zona IV del AUDIT y III del DAST, sus respuestas a las preguntas abiertas fueron 1 . ¿Consideras que tienes algún problema con el consumo de bebidas alcohólicas o drogas? ¿Por qué? $R=$ No, porque las consumo, pero soy una persona funcional 2. ¿Consideras que en tu escuela hay un problema de consumo de bebidas alcohólicas y/o drogas? ¿Por qué? No, la mayoría las observo saludables.3. ¿Participarías en una investigación o tratamiento para disminuir el consumo de bebidas alcohólicas y/o drogas? ¿Por qué? Sí, me gustaría conocer esa dinámica de la comunidad de la ESAT en especial.

\section{Discusión}

Con los resultados obtenidos podemos afirmar que no hay un consumo elevado ni frecuente en los estudiantes la Escuela Superior de Atotonilco de Tula. Sin embargo, las cifras indican que es necesario implementar programas de prevención, educación y consejería para evitar que el consumo que tienen los estudiantes llegue a niveles perjudiciales. Así mismo, es importante tomar en cuenta que aunque en general no se observa una situación alarmante hay casos particulares que pudiesen requerir atención especial. Desafortunadamente el anonimato impide conocer que estudiantes pudiesen requerir atención especial, sin embargo, resultaría relevante dar a conocer estos resultados dentro de la ESAT e informales a los jóvenes de los programas de atención psicopedagógica con los que cuenta la escuela. Es importante recalcar la apertura que tuvieron los estudiantes para contestar las preguntas, todos se mostraron participativos e interesados en el estudio, muestra de ellos es que más de la mayoría participaría en más investigaciones al respecto de las adicciones e incluso en intervenciones de ser necesario.

Finalmente, agradecemos a las autoridades de la Escuela Superior de Atotonilco de Tula, quienes otorgaron el permiso para que esta investigación se llevara a cabo. $Y$ 
estamos seguros que tomaran con interés el reporte aquí presentado.

\section{Referencias}

1. Frg F, Lj P, Lr A, Ksl G, See A. Trastornos por consumo de alcohol (AUDIT) en adolescentes y jovenes marginales de bandas juveniles de mexico. Esc Ana Nery Rev Enferm. 2007;11(4):8.

2. Instituto Nacional de Psiquiatría Ramón de la Fuente Muñiz, Instituto Nacional de Salud Pública CN contra las A, Secretaría de Salud. Encuesta Nacional de Consumo de Drogas, Alcohol y Tabaco 2016-2017: Reporte de Alcohol [Internet]. México: INPRFM; 2017. Disponible en:

https://encuestas.insp.mx/ena/encodat2017/encodat_drogas_20 16_2017.pdf

3. Instituto Nacional de Psiquiatría Ramón de la Fuente Muñiz, Instituto Nacional de Salud Pública CN contra las A, Secretaría de Salud. Encuesta Nacional de Consumo de Drogas, Alcohol y Tabaco 2016-2017: Reporte de Drogas [Internet]. México: INPRFM; 2017. Disponible en:

https://encuestas.insp.mx/ena/encodat2017/encodat_drogas_20 16_2017.pdf

4. Emilia Lucio Gómez-Maqueo, Hugo Leonardo Gómez Hernández, Brenda Morales Rodríguez, Marisol Pérez Ramos. Uso del AUDIT y el DAST-10 para la identificación de abuso de sustancias psicoactivas y alcohol en adolescentes. Rev Colomb Psicol. 2009;18(1):9-17. 